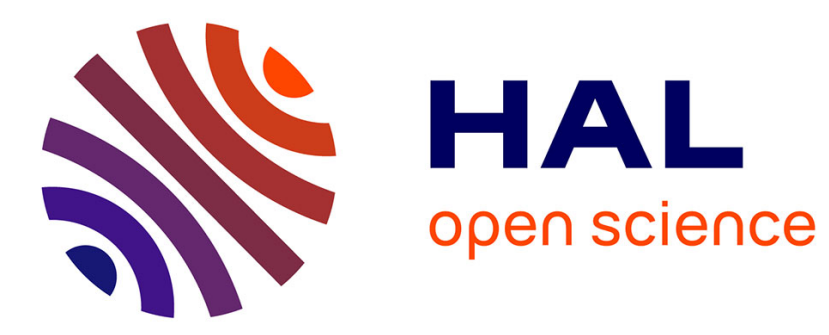

\title{
Intention et réalisation de migration de retour au Sénégal et en République démocratique du Congo
}

Marie-Laurence Flahaux

\section{To cite this version:}

Marie-Laurence Flahaux. Intention et réalisation de migration de retour au Sénégal et en République démocratique du Congo. Population (English edition), 2015, pp.103-133. 10.3917/popu.1501.0103 . hal-01872649

\section{HAL Id: hal-01872649 \\ https://hal.science/hal-01872649}

Submitted on 21 Apr 2021

HAL is a multi-disciplinary open access archive for the deposit and dissemination of scientific research documents, whether they are published or not. The documents may come from teaching and research institutions in France or abroad, or from public or private research centers.
L'archive ouverte pluridisciplinaire HAL, est destinée au dépôt et à la diffusion de documents scientifiques de niveau recherche, publiés ou non, émanant des établissements d'enseignement et de recherche français ou étrangers, des laboratoires publics ou privés. 


\title{
Migration de retour au Sénégal et en RD Congo : intention et réalisation
}

\author{
Marie-Laurence Flahaux
}

FLAHAUX, M.-L. (2015). "Intention et réalisation de migration de retour au Sénégal et en République démocratique du Congo ", Population, 70(1), 103-133, DOI:10.3917/popu.1501.0103.

\section{Résumé}

A partir des données biographiques du projet MAFE qui ont été collectées à la fois auprès de migrants pendant leur séjour en Europe et de migrants de retour dans leur pays d'origine, cet article vise à analyser, d'une part, l'intention initiale de retour des Sénégalais et des Congolais qui ont migré en Europe et, $d$ 'autre part, la réalisation de leur intention de retour. Les résultats révèlent que, au moment de leur arrivée, les individus envisagent de retourner dans leur pays d'origine s'ils ont migré en Europe avec l'objectif d'acquérir des ressources qu'ils pourront valoriser dans leur pays après leur retour. Cependant, si la situation dans le pays d'origine se dégrade fortement, comme c'est le cas en République démocratique du Congo à partir des années 1990, les migrants sont davantage dans l'optique de s'établir définitivement en Europe. En outre, plus il est difficile de migrer en Europe et moins les migrants ont l'intention de retourner car ils savent que, s'ils retournent, ils ne pourront pas revenir facilement en Europe en cas d'échec ou de problème dans leur pays. Les analyses suggèrent enfin que la détérioration du contexte dans les pays d'origine et les politiques migratoires restrictives découragent le retour des migrants qui avaient pourtant, au moment de leur arrivée en Europe, l'intention de retourner dans leur pays d'origine.

FLAHAUX, M.-L. (2015). "Return migration to Senegal and DR Congo: intention and realisation", Population, 70 (1), 97-126, DOI : 10.3917/popu.1501.0103.

\begin{abstract}
Using life event history data collected by the MAFE project survey of migrants in Europe and return migrants in their countries of origin, this article aims to analyse, first, the initial return intentions of Senegalese and Congolese migrants to Europe, and second, the realization of those intentions. The results reveal that at the time of their arrival, individuals who migrate to Europe with the objective of acquiring resources for later use in their country of origin plan to return there. However, if the situation in their country of origin seriously deteriorates, as was the case in the Democratic Republic of Congo from the 1990s onward, migrants tend to plan to remain permanently in Europe. Furthermore, the more difficult it is to migrate to Europe, the less likely migrants are to plan to return. Finally, worsening political and economic conditions in the origin country and restrictive immigration policies in host countries discourage migrants who initially plan to return home from following through on those plans.
\end{abstract}




\section{Introduction}

Bien que la question du retour des migrants ait fait l'objet d'un nombre croissant d'études empiriques, en particulier dans la dernière décennie (Carling, Mortensen et $\mathrm{Wu}, 2011$ ), certains aspects n'ont pas ou n'ont été que très peu étudiés. En particulier, l'intention initiale des migrants quant au retour et l'éventuelle concrétisation de leur intention n'a pas été abordée en profondeur dans la littérature théorique et empirique des migrations internationales.

Comme le soulèvent de Haas et Fokkema (2011), les théories économiques classiques des migrations internationales présentent le désavantage de lier le retour des migrants au motif initial de leur migration. Ainsi, pour la théorie néoclassique, qui considère les migrants comme des acteurs rationnels qui migrent sur base d'un calcul de coûts et bénéfices pour maximiser leurs revenus (Todaro, 1969), la migration a lieu dès le départ dans une optique définitive. Dès lors, le retour est perçu comme étant la conséquence d'un échec à l'étranger car les bénéfices que les migrants attendaient de la migration n'ont pas été atteints (Cassarino, 2004 ; Constant et Massey, 2002). Pour la théorie de la nouvelle économie de la migration de travail, la migration est une des stratégies que les ménages adoptent pour diversifier les sources de revenus dans un contexte caractérisé par la défaillance des marchés (accès au crédit, aux assurances) (Stark et Bloom, 1985). Selon cette théorie, les migrants sont dès le départ dans l'optique de retourner dans leur pays d'origine lorsqu'ils auront réalisé à l'étranger un objectif bien précis : celui d'acquérir les ressources nécessaires pour surmonter les imperfections des marchés, ce qui leur permettra d'améliorer leurs conditions de vie et celles de leur famille. Il est considéré que les migrants qui retournent sont ceux qui ont acquis assez de capital financier et humain pour pouvoir réaliser leurs projets dans le pays d'origine (Cassarino, 2004). Ces deux théories ne tiennent pas compte du fait que les motivations initiales et les stratégies des migrants peuvent évoluer pendant leur séjour à l'étranger (de Haas et Fokkema, 2011). Si certains migrants retournent comme ils le prévoyaient au départ, d'autres vont finalement préférer rester à l'étranger, car les liens avec leur communauté d'origine se sont atténués (Guilmoto et Sandron, 2000 ; Massey, Alarcon, Durand et Gonzalez, 1987), parce qu'ils ont acquis une certaine autonomie (El Hariri, 2003), parce que des événements d'ordre personnel ou professionnels sont survenus dans leur vie (Hazen et Alberts, 2006), ou en raison d'un changement de contexte dans leur pays d'origine ou de destination (Carling, 2004 ; Massey, Durand et Malone, 2002 ; Cornelius, 2001 ; Massey et Espinosa, 1997). Pour d'autres, le projet de retour reste présent au fil du temps, mais est remis continuellement à plus tard, si bien qu'il prend la forme d'un mythe (Sayad, 1998 ; El Hariri, 2003).

En raison de l'absence de données, rares sont les travaux quantitatifs qui se sont spécifiquement intéressés à l'intention initiale des migrants quant au retour et à leur réalisation. D'une part, les recherches s'intéressant à l'intention de retour des migrants se basent sur des enquêtes transversales qui posent la question de l'intention de retour au moment de l'enquête, quelle que soit la durée depuis laquelle ils sont arrivés dans le pays de destination, et non au moment de leur arrivée. D'autre part, l'analyse de la réalisation du retour suppose des données sur les histoires de vie des individus qui sont retournés ainsi que sur celles de ceux qui sont toujours à l'étranger. Jusqu'à récemment, seul le Mexican Migration Project, à partir de données collectées à la fois dans les pays d'origine et de destination, a permis d'étudier les facteurs déterminants le retour des Mexicains qui ont migré aux Etats-Unis (Reyes, 2004 ; Massey et Espinosa, 1997). 
A partir des enquêtes biographiques MAFE-Sénégal et MAFE-Congo qui ont été réalisées dans des pays d'origine en Afrique et de destination en Europe et qui ont collecté des informations à la fois sur les intentions initiales de retour des migrants et sur les retours, cet article poursuit deux objectifs. II vise à analyser (1) le projet migratoire des Sénégalais et des Congolais au moment de leur arrivée en Europe, et (2) la concrétisation de leur intention initiale de retour.

L'hypothèse posée est que le projet migratoire initial et le retour dépendent de ce que les migrants estiment être le mieux pour eux et pour leur famille, mais que ces aspirations sont limitées par la situation prévalant dans les pays d'origine et de destination. On s'attend à ce qu'une détérioration du contexte dans les pays d'origine et un durcissement des politiques migratoires édictées par les pays de destination aient un effet négatif sur l'intention de retour des individus au début de leur migration. Il est aussi attendu que l'intention initiale quant au retour ne se traduise pas en retour si les restrictions pour migrer s'intensifient et si la situation dans les pays d'origine se détériore. A ce titre, le Sénégal et la République démocratique du Congo (RD Congo) présentent deux contextes intéressants à comparer car ils ont connu des évolutions politiques et économiques différentes.

La première section de cet article consiste en une brève revue de littérature sur les déterminants de l'intention et du retour des migrants. La deuxième section retrace l'évolution du contexte au Sénégal et en RD Congo ainsi que des politiques migratoires en Europe, avant de présenter l'histoire et la place du retour dans les migrations sénégalaises et congolaises. Dans une troisième section, les hypothèses de recherche sont détaillées. Enfin, la quatrième section expose la méthodologie de recherche, et la cinquième section présente les résultats.

\section{Revue de littérature sur les déterminants de l'intention et de la réalisation du retour}

La littérature met en avant de nombreux facteurs influençant l'intention et le retour des migrants. ${ }^{1}$ Cette revue de littérature se focalise particulièrement sur les facteurs reflétant les aspirations des migrants (motif de la migration) et sur les contraintes qui peuvent s'imposer à eux (statut administratif, politiques migratoires restrictives et détérioration de la situation dans le pays d'origine).

Le motif du départ à l'étranger apparaît comme un déterminant fort. Les individus qui ont migré pour des raisons familiales s'installent généralement dans le pays de destination avec l'intention d'y rester, comme le montre une étude sur les migrants afghans, burundais, éthiopiens et marocains aux Pays-Bas (Bilgili et Siegel, 2012). Une autre étude sur les PaysBas confirme que, dans les faits, le regroupement familial donne souvent lieu à une migration définitive (Bijwaard, 2007). Concernant les migrations pour motif d'études, il semble qu'elles ont plus souvent lieu dans une optique temporaire, en particulier si les conditions économiques y sont favorables (Baruch, Budhwar et Khatri, 2007). Une recherche montre cependant que les étudiants africains aux Etats-Unis souhaitent retourner dans leur pays d'origine autant que les autres étudiants étrangers car ils savent qu'ils trouveront un emploi relativement facilement à leur retour (Hazen et Alberts, 2006). Les individus qui ont quitté leur pays pour des raisons politiques semblent être les plus enclins à rester définitivement à l'étranger, comme le révèlent des études sur le cas de la Suède (Edin, Lalonde et Aslund, 2000) et de la Norvège (Carling et Pettersen, 2014).

\footnotetext{
${ }^{1}$ Pour une analyse détaillée de la littérature, voir Flahaux, 2013, p. 107-131.
} 
Le statut administratif ou légal des migrants dans le pays de destination semble aussi être important pour le retour. Selon Reyes (2004), les migrants en situation irrégulière ne retournent pas au Mexique mais restent aux Etats-Unis dans l'espoir que leur statut soit régularisé. Les travaux qualitatifs de Sinatti (2011) vont dans le même sens à propos des Sénégalais en situation irrégulière en Italie. Dans la même lignée, des recherches sur les migrations capverdiennes (Carling, 2004), marocaines (de Haas et Fokkema, 2010) et mexicaines (Cornelius, 2001 ; Massey et Espinosa, 1997 ; Massey, Durand et Malone, 2002 ; Reyes, 2004) suggèrent que les individus peuvent différer ou annuler leur projet de retour en raison des politiques migratoires de plus en plus restrictives dans les pays de destination.

Enfin, la situation prévalant dans les pays d'origine apparait aussi comme un facteur crucial. Des études réalisées sur le cas du Maroc (de Haas et Fokkema, 2010 et 2011) et de l'Amérique latine (Moran-Taylor et Menjívar, 2005) ont mis en avant que les migrants envisagent souvent de ne pas retourner lorsqu'ils ont quitté un contexte instable et insécurisé ; dans les faits, les probabilités de retour sont aussi plus faibles quand le pays d'origine connaît une crise, car les migrants appréhendent le manque d'opportunités d'emploi et le climat politique défavorable.

\section{Contextualisation de la recherche}

\section{Le Sénégal et la RD Congo, des pays aux évolutions politico-économiques contrastées}

Le Sénégal et la RD Congo ont connu des évolutions très différentes sur le plan politique depuis leur indépendance. Anciennement colonisé par la France, le Sénégal est réputé pour être l'un des pays les plus stables du continent africain. Cela est attesté par son alternance politique au pouvoir et par sa capacité à surmonter les crises (Dumont et Kanté, 2009). L'histoire de la RD Congo, colonisée jusqu'en 1960 par la Belgique, a connu des crises politiques plus profondes. Mobutu, commandant en chef de l'armée, est resté au pouvoir 32 années, caractérisées par une relative stabilité du fait de l'instauration d'un régime politique très centralisé et répressif. L'échec du processus de démocratisation amorcé au début des années 1990 a plongé le pays dans une longue période de troubles politiques, donnant lieu à des émeutes, des mutineries de l'armée et des pillages sans précédent (Braeckman, 2009). Une lente transition vers le changement est apparue en 1997, lorsque Mobutu a été chassé du pouvoir par L.D. Kabila, mais les violences ont persisté jusqu'à l'assassinat de ce dernier en 2001 (Hesselbein, 2007). En 2006, les premières élections ont été organisées. J. Kabila a été élu président et a été réélu en 2011, bien que ce résultat ait été contesté par une partie de la population, en particulier à Kinshasa. ${ }^{2}$

Sur le plan économique, les populations sénégalaises et congolaises ont vu leurs conditions de vie se dégrader au fil du temps, notamment suite à la mise en place des programmes d'ajustements structurels et du retrait de l'Etat des secteurs sociaux (Thioub, Diop et Boone, 1998 ; Braeckman, 2009). Au Sénégal, l'impact de la crise s'est fait sentir à partir des années 1990 sur le marché de l'emploi. A Dakar, le chômage et les emplois informels se sont développés, et il est devenu particulièrement difficile pour les individus les plus instruits d'obtenir un emploi salarié qualifié (Bocquier, 1996). Du côté congolais, la détérioration de la situation politique a contribué grandement à la dégradation du contexte économique. Les premières années de la décennie 1990 ont constitué la période la plus sombre de l'histoire

\footnotetext{
${ }^{2}$ Kinshasa, la capitale, située à l'ouest du pays, a été relativement tenue à l'écart de ces conflits armés qui ont déchiré la RD Congo, surtout par rapport à l'est du pays.
} 
du pays. A cette période, la RD Congo a vu le taux de croissance de son PIB chuter fortement, l'inflation augmenter, et la dévaluation de la monnaie s'accélérer (Peemans, 1998). Les répercussions se sont fait fortement ressentir par la population. Ce n'est qu'au début des années 2000 que le pays a connu une timide reprise économique et que sa population a constaté une légère amélioration des conditions de vie (Mangalu, 2011). En 2014, la RD Congo occupe l'avant-dernière place dans le classement international des pays en fonction de leur développement humain.

\section{Des politiques migratoires tournées vers d'avantage de restrictions en Europe}

Les politiques migratoires qui règlementent l'accès et le séjour des étrangers sur le territoire des pays européens ont considérablement évolué au cours du temps. Migrer en Europe à partir des pays tiers et y séjourner est devenu globalement de plus en plus difficile, bien que les politiques aient varié selon les pays, l'origine et le type de migrants.

Dans les années 1960, plusieurs pays européens ont fait appel à la main-d'œuvre étrangère dans le contexte de la croissance économique. A cette époque, les conditions pour migrer étaient souples (Donovon, 1988). La France a pris des dispositions pour encourager la libre circulation de la main-d'œuvre originaire de ses anciennes colonies et a mis en place des bureaux de recrutement, entre autres au Sénégal (Robin, Lalou et Ndiaye, 2000). La Belgique n'a jamais procédé au recrutement de travailleurs congolais, mais a octroyé aux ressortissants de son ancienne colonie des visas et des bourses d'études dans des universités belges dans le but de créer une élite qui pourrait se porter garante de la gestion du pays nouvellement indépendant (De Schutter, 2011). Entre 1973 et 1974, avec la crise économique, des mesures ont été prises pour mettre fin au système de recrutement de la main-d'œuvre (Castles, 2006). II n'a dès lors plus été possible de migrer pour un motif de travail, mais le titre de séjour lié au regroupement familial a été créé. L'entrée en France, pour les Sénégalais, a toutefois été facilitée grâce à l'exemption de visa de voyage jusqu'en 1986 (Marot, 1995), et en Italie jusqu'en 1990 (Finotelli et Sciortino, 2009). Le visa donnant accès au territoire belge a quant à lui toujours été obligatoire pour les ressortissants congolais (Geert, 2011).

Dans les années 1990, les migrations sont devenues un sujet de plus en plus politisé dans de nombreux pays d'Europe. Les conditions pour migrer dans un cadre familial, d'études ou de travail se sont durcies (Gnisci, 2008). A partir des années 2000, la lutte contre la migration irrégulière est devenue le champ prioritaire des actions au niveau européen (Guiraudon, 2000). Avec la création de l'agence FRONTEX, tout un arsenal ainsi que des moyens considérables ont été mis en place pour tenter d'empêcher l'accès au territoire européen aux migrants ne détenant pas de visa (Carling et Hernández-Carretero, 2011). Les procédures pour obtenir le statut de réfugié se sont durcies également, et l'accent a été mis sur le renvoi des migrants en situation irrégulière dans leur pays d'origine (Cassarino, 2008).

En raison de la rupture qui a eu lieu au début des années 1990 au niveau de la situation dans les pays d'origine et des politiques migratoires, la périodisation " avant " et " après " 1990 sera utilisée dans les analyses de cet article.

\section{La place du retour dans les migrations sénégalaises et congolaises}

Les évolutions contrastées que le Sénégal et la RD Congo ont connues ont influencé les histoires migratoires de leurs populations, y compris les retours. Les études quantitatives qui 
ont analysé l'importance des intentions de retour et des retours sont rares; ce sont surtout des travaux qualitatifs qui apportent des éclairages sur la question.

\section{a) La place du retour dans les migrations sénégalaises}

A l'origine, la migration sénégalaise en Europe était principalement celle d'hommes seuls, issus du milieu rural, qui allaient travailler dans I'industrie en France (Timera, 1996). Ils effectuaient des aller-et-retour entre la France et le Sénégal avant de retourner définitivement au pays, moment auquel ils étaient remplacés par d'autres membres de leur communauté, selon le système de la noria (Barou, 2001). Cependant, après 1974, lorsque les Etats européens ont entrepris de réguler leurs frontières, ces migrations de remplacement ont pris fin (Barou, 2001), et certains migrants sénégalais ont fait le choix de recourir au regroupement familial dans le pays de destination (Azoulay et Quiminal, 2002). Des travaux qualitatifs soulignent que le retour est resté au cœur du projet migratoire des Sénégalais. Selon le point de vue sénégalais, en effet, une migration réussie donne lieu à un retour définitif auprès de la famille, après en avoir amélioré les conditions de vie (Sinatti, 2011). Ceci dit, le retour est souvent remis à plus tard, les migrants étant confrontés aux pressions familiales et à la difficulté d'investir leur épargne dans des projets personnels au Sénégal (Hernandez Carretero, 2012). Cependant, les données de l'enquête " Push Pull ", réalisée en 1997 auprès de Sénégalais en Italie et en Espagne, révèlent que seuls 38\% d'entre eux ont l'intention de retourner au Sénégal, 31\% entendant rester en Europe et les autres étant indécis (de Haas et Fokkema, 2011).

\section{b) La place du retour dans les migrations congolaises}

C'est à partir de 1960 que les Congolais ont commencé à migrer vers l'Europe, essentiellement en Belgique dans un cadre d'études (Kagné et Martiniello, 2001 ; Schoonvaere, 2010). A cette époque, bien que tenant sur des pieds fragiles, l'Etat congolais pourvoyait à des services sociaux, et les migrants retournaient au pays après leurs études (Sumata, Trefon et Cogels, 2004). Ils se voyaient offrir un emploi de cadre, un bon salaire et des conditions de vie favorables dans leur pays d'origine (De Schutter, 2011). Cependant, par la suite, lorsque la situation s'est détériorée en RD Congo, l'installation des Congolais à l'étranger est devenue plus durable. Comme l'expliquent Bongo-Pasi Moke Sangol et Tsakala Munikengi (2004), avant, les familles congolaises dont les enfants étudiaient en Europe et en Amérique du Nord les encourageaient à achever rapidement leurs études et à rentrer au pays; aujourd'hui, en revanche, elles les poussent à rester à l'étranger à tout prix et à y trouver un emploi, quel qu'il soit, de façon à ce qu'ils pourvoient aux besoins des parents restés en RD Congo. Bazonzi (2010) écrit : " Une fois en terre étrangère, l'on est prêt à tout faire, sauf retourner au pays, de peur d'être la risée du voisinage " (Banzonzi, 2010, p. 8). L'étude quantitative de Schoonvaere (2010), réalisée à partir des données du Registre national belge, révèle également que les migrations des Congolais en Belgique sont devenues plus permanentes au fil du temps : parmi ceux arrivés en Belgique en 1991, 18\% étaient déjà repartis de Belgique au bout de deux ans alors que, dix ans plus tard, la proportion n'était plus que de $8 \%$.

\section{Objectifs et hypothèses de recherche}

Dans un premier temps, cette recherche vise à analyser les facteurs explicatifs de l'intention initiale de retour des migrants sénégalais et congolais qui ont migré en Europe. On s'attend à ce que les migrants envisagent initialement de retourner s'ils ont migré dans le but 
d'acquérir des ressources qu'ils pourront valoriser dans leur pays d'origine par la suite. Par contre, il est possible que la détérioration du contexte dans les pays d'origine, en particulier pour les Congolais, les poussent à migrer dans une optique définitive. On s'attend aussi à ce que les migrants qui ont eu des difficultés pour migrer en raison des politiques migratoires restrictives n'aient pas l'intention de retourner dès le début de leur séjour en Europe. Il est en effet possible que ces migrants ne désirent pas prendre le risque de retourner car ils savent qu'il leur sera difficile de migrer à nouveau par la suite, en cas de réinsertion difficile au pays.

Dans un deuxième temps, on cherche à savoir si l'intention initiale de retour des migrants donne lieu à un retour. L'objectif, en particulier, est de répondre à la question : quels sont les migrants qui ne réalisent pas un retour alors qu'ils l'avaient prévu, et comment peut-on l'expliquer? On pose I'hypothèse que l'intention initiale de retour est généralement un déterminant fort des retours, mais que les politiques migratoires restrictives et la détérioration de la situation dans les pays d'origine ont pour effet d'annuler ou de retarder la réalisation de l'intention de retour de certains migrants, surtout des Congolais, et dans une moindre mesure des Sénégalais, en raison de la crise moins sévère qui a sévi au Sénégal par rapport à la RD Congo.

Le tableau 1 spécifie les hypothèses de recherche.

Tableau 1. Les hypothèses spécifiques de recherche, par facteur susceptible d'influencer l'intention initiale de retour et sa réalisation

\begin{tabular}{|l|l|l|}
\hline Type de facteurs & \multicolumn{1}{|c|}{ Intention initiale de retour } & Réalisation de l'intention initiale de retour \\
\hline $\begin{array}{l}\text { H1 : Motif de } \\
\text { départ }\end{array}$ & $\begin{array}{l}\text { H1a : Migrants des deux pays partant pour } \\
\text { acquérir des ressources valorisables au pays } \\
\text { plus susceptibles de vouloir retourner (cas } \\
\text { des migrations pour raison d'études et } \\
\text { professionnelles), ce qui n'est pas attendu } \\
\text { pour ceux qui migrent dans un cadre } \\
\text { familial ou pour une raison politique. }\end{array}$ & $\begin{array}{l}\text { H1b : Sénégalais susceptibles de réaliser } \\
\text { leur intention initiale, mais pas les } \\
\text { Congolais, vu la détérioration du contexte } \\
\text { dans leur pays d'origine. }\end{array}$ \\
\hline $\begin{array}{l}\text { H2 : Situation } \\
\text { administrative }\end{array}$ & $\begin{array}{l}\text { H2a: Migrants des deux pays en situation } \\
\text { irrégulière au début de la migration moins } \\
\text { susceptibles de vouloir retourner. }\end{array}$ & $\begin{array}{l}\text { H2b : Migrants des deux pays en situation } \\
\text { irrégulière moins susceptibles de retourner. }\end{array}$ \\
\hline $\begin{array}{l}\text { H3: Provenance } \\
\text { directe du pays } \\
\text { d'origine }\end{array}$ & $\begin{array}{l}\text { H3a : Migrants des deux pays n'ayant pas } \\
\text { migré directement en Europe moins } \\
\text { susceptibles de vouloir retourner. }\end{array}$ & $\begin{array}{l}\text { H3b : Migrants des deux pays n'ayant pas } \\
\text { migré directement en Europe moins } \\
\text { susceptibles de retourner. }\end{array}$ \\
\hline $\begin{array}{l}\text { H4 : Période et } \\
\text { intention }\end{array}$ & $\begin{array}{l}\text { H4a: Congolais partis après 1990 moins } \\
\text { susceptibles de vouloir retourner ; pas de } \\
\text { différence pour les Sénégalais. }\end{array}$ & $\begin{array}{l}\text { H2b : Avant 1990, migrants des deux pays } \\
\text { ayant l'intention de retourner plus } \\
\text { susceptibles de retourner que ceux } \\
\text { n'envisageant pas de retour. Après 1990, } \\
\text { migrants des deux pays ayant l'intention de } \\
\text { retourner pas plus susceptibles de } \\
\text { retourner. }\end{array}$ \\
\hline
\end{tabular}

\section{Méthodologie}

\section{Données}

Les données des enquêtes biographiques du projet MAFE présentent des avantages pour étudier la question du retour au Sénégal et en RD Congo. Ces enquêtes ont été réalisées à la fois dans les pays d'origine des migrants (dans les régions de Dakar et de Kinshasa) et dans plusieurs pays de destination en Europe (en France, Italie et Espagne pour les Sénégalais, et 
en Belgique et au Royaume-Uni pour les Congolais) ; elles ont collecté de façon rétrospective des données sur les histoires de vie de migrants et de migrants de retour. De ce fait, on peut connaître la situation des individus au moment de leur arrivée en Europe et pendant toute leur migration. Les questionnaires identiques qui ont été utilisés dans les différents pays permettent des analyses comparatives entre les migrants sénégalais et congolais. L'échantillonnage et la nature de ces enquêtes comportent néanmoins certaines limites.

Les analyses sur la question du retour des migrants au Sénégal et en RD Congo ne sont d'abord pas représentatives de tous les Sénégalais et de tous les Congolais qui ont migré en Europe, puisque les enquêtes n'ont pas été réalisées dans tous les pays européens. Les enquêtes ne sont pas représentatives des pays d'origine, au niveau national, car elles ne se sont déroulées que dans les régions des capitales, Dakar et Kinshasa. Toutefois, tous les migrants enquêtés ne sont pas originaires de ces régions ${ }^{3}$. Bien que cette limite appelle à la prudence dans la généralisation des résultats, le fait que la majorité des migrants aient déjà vécu dans la capitale de leur pays et les efforts de représentativité qui ont été faits dans les plans d'échantillonnage permettent de faire l'hypothèse que les migrants qui n'ont jamais vécu dans les régions du pays d'origine où ont eu lieu les enquêtes ne sont pas radicalement différents de ceux qui y ont vécu. Une autre limite à soulever est celle des faibles effectifs qui caractérisent les échantillons de migrants et surtout de migrants de retour. Malgré le sur-échantillonnage des migrants de retour dans les enquêtes qui se sont déroulées dans les pays d'origine, le nombre de migrants de retour d'Europe n'est pas très élevé. Cela reflète la relativement faible propension au retour des migrants. Afin d'avoir le plus de retours possible dans les échantillons, le choix a été fait d'inclure dans les analyses tous les retours que les migrants ont pu effectuer, quels que soient les pays européens où ils ont séjourné.

D'autres limites sont liées à la nature des enquêtes biographiques. Les efforts de mémoire que les répondants doivent faire pour se rappeler de leur passé peuvent être considérables. Bien que les questionnaires aient été conçus pour favoriser les souvenirs des migrants, des erreurs peuvent survenir lorsque les personnes enquêtées oublient de déclarer certains événements, les déclarent partiellement ou les datent mal. Les informations relatives à des intentions passées, telles que l'intention de retour au moment de l'arrivée en Europe, peuvent être difficiles à se remémorer pour les répondants. Les individus réélaborant souvent leur projet migratoire en fonction de leur situation et de leurs interlocuteurs, il est possible que les réponses soient influencées par l'intention actuelle des répondants - qui ont pu évoluer depuis leur arrivée - ou par le fait qu'ils sont retournés (rationalisation a posteriori). Les questions - ouvertes - sur les motifs du départ et du retour sont également obtenues rétrospectivement et des biais peuvent être présents avec l'effet du temps.

\section{a) L'intention initiale de retour et le retour}

Le questionnaire ne contient pas de question précise sur l'intention de retour des migrants au moment où ils ont quitté leur pays d'origine. La variable sur l'intention initiale de retour est construite à partir de plusieurs questions posées à propos du séjour dans le premier pays européen dans lequel les migrants arrivent ${ }^{4}$ :

\footnotetext{
${ }^{3}$ Pour de plus amples détails sur les données du projet MAFE, voir le chapitre introductif de ce numéro.

${ }^{4}$ Dans les enquêtes MAFE, il est considéré qu'il y a migration dès lors qu'un séjour de plus d'un an en Europe a été effectué. Les séjours de moins d'un an ne sont pas pris en compte.
} 
- "Au départ, combien de temps comptiez-vous rester dans ce pays de séjour ? ". Les individus enquêtés répondent à cette question en donnant un nombre défini d'années ou en affirmant " définitivement ". Certains répondent qu'ils ne savent pas.

- "Quand vous êtes arrivé dans ce pays de séjour, (1) vous considériez qu'il s'agissait de votre destination finale, celle où vous aviez prévu d'aller au départ; (2) vous n'aviez pas d'idée précise sur le pays où vous vouliez aller; (3) vous aviez en tête d'aller ailleurs, il s'agissait donc d'un pays intermédiaire ".

- Lorsque les enquêtés déclarent qu'ils avaient en tête d'aller ailleurs (3), les questions suivantes leur sont posées: "Quel pays aviez-vous en tête de rejoindre ensuite ? ", "Et dans quel pays souhaitiez-vous arriver tout à la fin ? ". A cette dernière question, les migrants donnent le nom d'un autre pays, mais certains déclarent que leur destination finale est leur pays d'origine.

Partant de ces informations, la variable sur l'intention initiale de retour est construite selon le schéma de la figure 1.

Figure 1. Construction de la variable sur l'intention initiale des migrants quant au retour

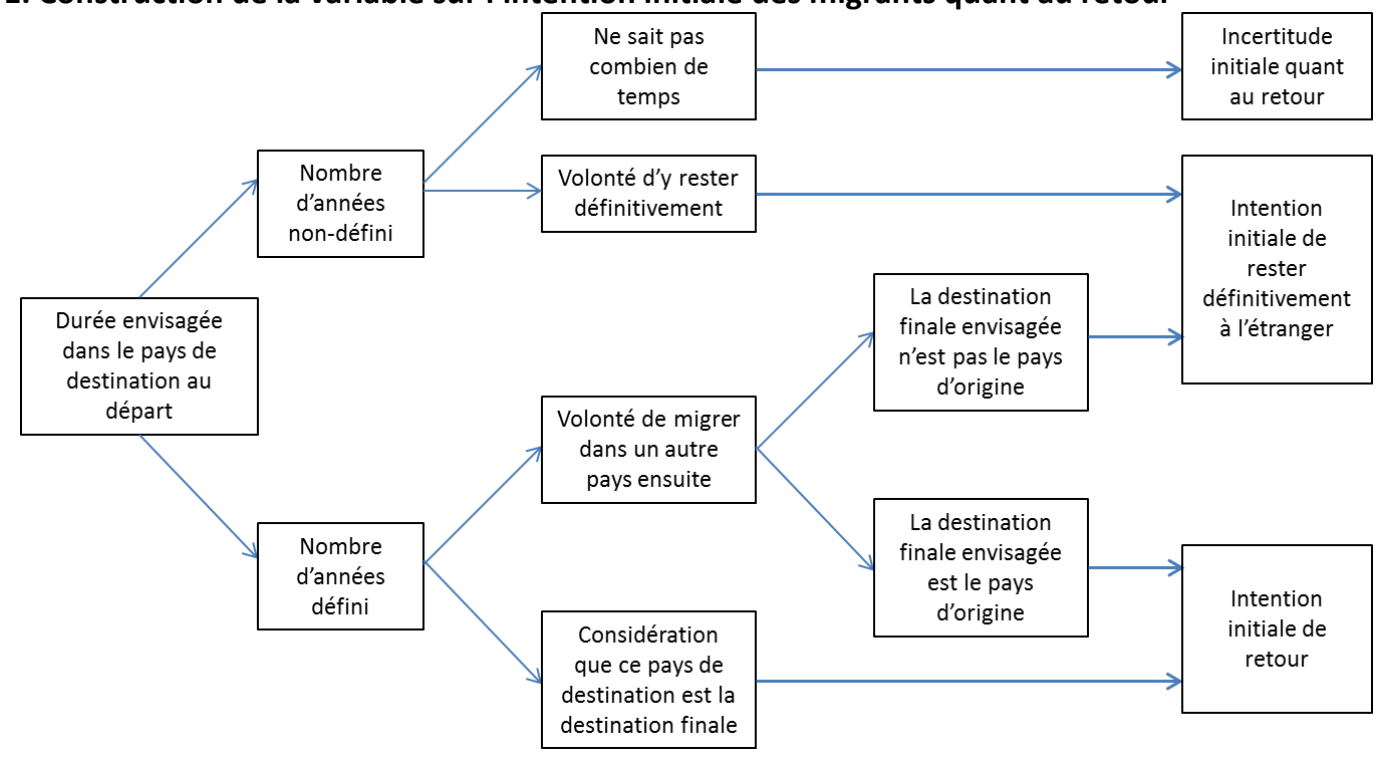

La variable sur l'intention initiale de retour distingue trois possibilités :

- Si les migrants déclarent vouloir rester pendant une durée précise dans le pays de destination, qu'ils estiment que ce pays constitue la destination finale de leur migration ou qu'ils envisagent de migrer ensuite ailleurs tout en ayant en tête de retourner dans leur pays d'origine à la fin, on considère qu'ils ont initialement I'intention de retourner.

- A contrario, si les individus déclarent vouloir s'installer définitivement dans le pays de destination ou avoir en tête de migrer ensuite ailleurs sans que la destination finale envisagée ne soit leur pays d'origine, on considère qu'il n'y a pas d'intention initiale de retour.

- Dans le cas où les migrants ne donnent pas de réponse à la question sur la durée pendant laquelle ils envisagent de rester dans le pays de destination, on considère qu'ils étaient incertains quant au retour.

Ensuite, on considère qu'il y a retour lorsque les migrants effectuent un mouvement de retour dans leur pays d'origine pour une durée de plus d'un an, ou de moins d'un an à condition qu'ils aient l'intention, au moment de ce retour, de s'y installer. On s'intéresse donc particulièrement aux retours ayant lieu dans une perspective de long terme; de ce fait, 
les retours qui ont lieu dans une optique temporaire, dans le cadre d'un séjour de vacances ou d'affaires, ne sont pas considérés comme des retours en tant que tels.

\section{b) Les variables explicatives d'intérêt}

Les enquêtes MAFE renseignent sur les caractéristiques socio-démographiques des individus tout au long de leur trajectoire de vie. Ainsi, on dispose d'informations sur leur sexe, sur leur âge chaque année depuis leur arrivée en Europe ainsi que sur leur niveau d'éducation, qui évolue en fonction des années qu'ils passent aux études. Des informations sur la situation individuelle des migrants sur le plan familial, matériel, professionnel et économique sont aussi disponibles pour chaque année passée en Europe. Ensuite, les enquêtes fournissent des informations relatives aux expériences migratoires. Ainsi, on peut connaître le pays dans lequel les individus ont migré, la durée et le rang de leur migration, mais aussi les circonstances dans lesquelles la migration a eu lieu. A cet égard, on dispose d'informations sur le motif de la migration et sur le fait que les individus sont arrivés en Europe en provenance directe de leur pays d'origine ou s'ils ont séjourné dans un pays tiers pendant au moins une année avant d'atteindre l'Europe. Enfin, les enquêtes contiennent des questions sur les visites que les migrants ont pu effectuer dans le pays d'origine depuis leur arrivée, et sur la situation administrative qu'ils ont dans le pays de destination. L'annexe 1 fournit des explications plus détaillées sur ces variables.

\section{Méthodes}

Les analyses sont divisées en deux parties. La première partie porte sur l'étude de l'intention initiale des migrants quant au retour. A cet égard, la situation des individus au moment de leur arrivée en Europe est considérée. Des régressions logistiques multinomiales simples sont réalisées pour analyser les déterminants de l'intention initiale de retour. Etant donné que l'on s'intéresse spécifiquement à expliquer l'intention initiale de retour par rapport à l'intention initiale de rester en Europe, les résultats sur les facteurs de l'incertitude quant au retour ne seront pas développés mais se trouvent dans l'annexe 2 .

La seconde partie traite de la question de la réalisation de l'intention de retour, ce qui nécessite de prendre en compte la durée de la migration en Europe, pendant laquelle les individus sont susceptibles de retourner. Les analyses prennent en considération le fait que, à côté des migrants qui ont effectué un retour dans leur pays d'origine, certains n'ont pas encore effectué de retour et d'autres ont quitté l'Europe pour une destination autre que leur pays d'origine. Les séjours en Europe de ces migrants partis ailleurs sont connus du fait qu'ils ont été enquêtés après leur retour dans leur pays d'origine ou en Europe lors d'une autre migration.

L'estimateur de Kaplan-Meier est utilisé pour calculer les risques pour les migrants de connaître l'évènement du retour au cours du temps selon la période de leur arrivée et leur intention initiale par rapport au retour. Pour analyser les déterminants du retour, des modèles biographiques en temps discret sont réalisés. Les variables explicatives sont mesurées pendant toute cette durée. Elles peuvent être fixes (comme le sexe ou le motif de la migration) ou varier dans le temps (comme l'âge, la situation administrative ou la période). Afin que les variables explicatives renvoient bien à la situation des migrants pendant cette durée d'exposition et non à leur situation après avoir connu l'événement, les événements du retour et du nouveau départ sont mesurés l'année ( $t-1)$ qui précède celle de l'événement du retour en $t$. Un même individu peut se retrouver plusieurs fois dans les échantillons s'il a connu plusieurs épisodes migratoires ou de retour. Etant donné qu'ils 
présentent des caractéristiques semblables lors de ces épisodes, on en tient compte pour le calcul des écarts-types; à cet égard, des grappes individuelles sont introduites dans les régressions. De plus, pour pallier les effectifs relativement faibles des échantillons, des techniques de réplication de type "Jackknife " sont utilisées pour chaque modèle. Elles permettent, à partir d'un seul échantillon, de générer de multiples échantillons (chaque échantillon étant égal au nombre d'observations moins une observation), sur la base desquels les écarts-types sont calculés (au lieu d'être calculés à partir de présomptions paramétriques). Ces méthodes sont dites "robustes" parce qu'elles sont moins dépendantes des valeurs extrêmes que les méthodes d'estimation classiques. Ces écartstypes fournissent généralement des intervalles de confiance plus larges que les estimateurs paramétriques. Cette méthode tend donc à rendre les résultats plus conservateurs, où seuls les résultats vraiment significatifs sont retenus. 


\section{Description des échantillons}

L'échantillon sénégalais comprend 713 migrations, tandis que l'échantillon congolais en compte 521. Les migrations de retour s'élèvent à 104 du côté sénégalais et à 86 du côté congolais. Les échantillons sont présentés dans le tableau 2. Il y apparaît notamment que le premier motif de migration des Sénégalais est l'amélioration de leurs conditions de vie, alors que les Congolais migrent d'abord pour des raisons d'études. Une majorité de migrants migre en Europe pour la première fois ${ }^{5}$, et respectivement $27 \%$ de Sénégalais et $16 \%$ de Congolais sont en situation irrégulière l'année de leur arrivée. Presque toutes les migrations ont lieu directement à partir du pays d'origine; seuls 6 ou 7\% des migrants ont séjourné au moins une année dans un pays tiers. $28 \%$ des migrants de l'échantillon sénégalais et $44 \%$ de migrants de l'échantillon congolais sont arrivés avant 1990. Les deux échantillons se différencient donc également sur ce point.

Tableau 2. Description des échantillons des migrants sénégalais et congolais l'année de leur arrivée en Europe (résultats pondérés)

\begin{tabular}{|c|c|c|c|}
\hline & & Sénégal & $\begin{array}{c}\text { RD } \\
\text { Congo }\end{array}$ \\
\hline Variables & Modalités & $\%$ & $\%$ \\
\hline \multirow{3}{*}{ Age } & $18-29$ ans & 54 & 63 \\
\hline & $30-44$ ans & 33 & 31 \\
\hline & 45 ans et plus & 4 & 6 \\
\hline \multirow{2}{*}{ Sexe } & Hommes & 70 & 59 \\
\hline & Femmes & 30 & 41 \\
\hline \multirow{2}{*}{ Niveau d'éducation atteint } & Aucun, primaire ou secondaire & 97 & 57 \\
\hline & Supérieur & 3 & 43 \\
\hline \multirow{5}{*}{ Motif de la migration } & Famille & 20 & 15 \\
\hline & Amélioration des conditions de vie & 53 & 20 \\
\hline & Etudes ou mission professionnelle & 18 & 45 \\
\hline & Raison politique & 1 & 14 \\
\hline & Autres & 8 & 6 \\
\hline \multirow{2}{*}{ Rang de la migration } & Première & 89 & 93 \\
\hline & Pas première & 11 & 7 \\
\hline \multirow{2}{*}{ Visite(s) } & Jamais de visite & 93 & 95 \\
\hline & Au moins une visite & 7 & 5 \\
\hline \multirow{3}{*}{$\begin{array}{l}\text { Intention initiale quant au } \\
\text { retour }\end{array}$} & Pas d'intention de retourner & 47 & 43 \\
\hline & Intention de retourner & 43 & 49 \\
\hline & Incertitude & 10 & 8 \\
\hline \multirow{3}{*}{ Situation administrative } & En situation régulière & 67 & 81 \\
\hline & En situation irrégulière & 27 & 16 \\
\hline & Valeur manquante & 6 & 3 \\
\hline \multirow{2}{*}{$\begin{array}{l}\text { Provenance directe du pays } \\
\text { d'origine }\end{array}$} & Oui & 94 & 93 \\
\hline & Non & 6 & 7 \\
\hline \multirow{2}{*}{ Destination } & Ancienne / traditionnelle & 52 & 47 \\
\hline & Nouvelle & 48 & 53 \\
\hline \multirow{5}{*}{ Situation familiale } & Famille dans pays d'origine & 38 & 28 \\
\hline & Famille dans pays de destination & 12 & 11 \\
\hline & Célibataire sans enfant & 40 & 43 \\
\hline & Famille dans pays de destination et d'origine & 7 & 18 \\
\hline & Famille ailleurs & 3 & 1 \\
\hline
\end{tabular}

\footnotetext{
${ }^{5}$ Dans les données sénégalaises, sur 713 migrations, 615 sont celles de Sénégalais qui migrent pour la première fois, 73 celles de ceux qui migrent pour la deuxième fois, 21 celles de ceux qui migrent pour la troisième fois, et 4 celles de ceux qui migrent pour la quatrième fois. Dans les données congolaises, sur 521 migrations, 412 sont celles de Congolais migrant pour la première fois, 90 celles de ceux migrant pour la deuxième fois, 15 celles de ceux migrant pour la troisième fois, et 4 celles de ceux migrant pour la quatrième fois.
} 


\begin{tabular}{|c|c|c|c|}
\hline \multirow{2}{*}{ Situation matérielle } & Propriétaire & 19 & 23 \\
\hline & Pas propriétaire & 81 & 77 \\
\hline \multirow{5}{*}{ Situation professionnelle } & Actif à hauteur de ses compétences & 45 & 4 \\
\hline & Actif en situation de sous-emploi & 19 & 18 \\
\hline & Inactif & 20 & 31 \\
\hline & Aux études & 16 & 45 \\
\hline & Valeur manquante & 0 & 1 \\
\hline \multirow{3}{*}{$\begin{array}{l}\text { Situation économique du } \\
\text { ménage }\end{array}$} & Moyens plus que suffisants & 17 & 34 \\
\hline & Moyens pas plus que suffisants & 83 & 66 \\
\hline & Valeur manquante & 1 & 0 \\
\hline \multirow{2}{*}{ Période } & Avant 1990 & 28 & 44 \\
\hline & A partir de 1990 & 72 & 56 \\
\hline Effectifs non-pondérés & & 713 & 521 \\
\hline
\end{tabular}

Source : Enquêtes biographiques MAFE-Sénégal (2008) et MAFE-Congo (2009).

\section{Résultats}

\section{L'intention initiale de retourner au pays}

Au moment de leur arrivée en Europe, moins de la moitié des migrants envisagent de retourner dans leur pays d'origine : $43 \%$ des Sénégalais et $49 \%$ pour les Congolais (tableau 2). La part de ceux qui ont initialement l'intention de retourner a diminué avec le temps, en particulier du côté congolais, où elle est passée de $64 \%$ parmi ceux qui sont arrivés en Europe avant 1990 à $36 \%$ parmi ceux qui y ont migré après cette date. Du côté sénégalais, les intentions initiales de retourner au pays sont également moins fréquentes à partir de 1990, mais elles n'ont diminué que dans une moindre mesure, passant de 51 à $40 \%$. La détérioration du contexte dans les pays d'origine explique cette diminution. En particulier, les crises politiques et économique en RD Congo qui ont marqué les années 1990 et 2000 ont engendré chez les migrants congolais le souhait de s'établir définitivement en Europe car ils ont perdu confiance en l'avenir de leur pays.

Lorsque l'on contrôle pour les caractéristiques des migrants et de leur expérience migratoire (tableau 3), aucune différence n'apparaît en termes d'intention initiale de retour entre les Sénégalais arrivés avant et après 1990. En revanche, les Congolais arrivés en Europe après 1990 ont cinq fois moins de chances de vouloir retourner en RD Congo par rapport à ceux qui ont migré avant 1990. Ce résultat, confirmant l'hypothèse $\mathrm{H} 4 \mathrm{a}$, montre que le début des années 1990 est un tournant dans les migrations congolaises. Si, avant 1990, les Congolais avaient une certaine confiance dans l'avenir de la RD Congo, la crise qui a sévi à partir de 1990 a été telle qu'ils n'ont, depuis, plus l'espoir que la situation se rétablisse. De ce fait, ceux qui ont l'opportunité de partir en Europe n'envisagent plus de retourner.

Les résultats montrent également que l'intention des migrants quant au retour dépend du motif de leur migration. Conformément à l'hypothèse $\mathrm{H} 1 \mathrm{a}$, les migrants des deux pays partant en Europe dans un cadre professionnel ou d'études présentent des chances très importantes de vouloir retourner : les Sénégalais en Europe pour cette raison sont 12 fois plus susceptibles d'avoir l'intention initiale de retourner par rapport à ceux qui migrent pour un motif familial, et les Congolais 13 fois. Ces migrants considèrent qu'ils auront des opportunités dans leur pays d'origine après la migration et qu'ils pourront valoriser à leur retour les compétences qu'ils auront acquises en Europe. En outre, les Sénégalais qui migrent pour améliorer leurs conditions de vie ont trois fois plus de chances d'avoir l'intention de retourner que ceux qui partent dans un cadre familial, mais cet effet n'apparaît pas pour les Congolais. Comme le suggère la littérature qualitative, les Sénégalais qui partent pour cette raison entendent retourner après avoir réalisé des économies qu'ils 
pourront investir au Sénégal (Sinatti, 2011 ; Hernandez Carretero, 2012). Le contexte stable du Sénégal permet aux migrants d'avoir un tel projet, mais celui-ci est moins envisageable pour les migrants originaires de RD Congo, où la situation est plus incertaine. Quant à ceux qui sont arrivés en Europe pour des raisons politiques, ils n'ont initialement ni plus ni moins le projet de retourner par rapport à ceux qui sont partis pour un motif familial.

Ensuite, les migrants des deux pays qui ne sont pas arrivés directement en Europe mais qui ont séjourné pendant au moins une année ailleurs auparavant sont davantage dans l'optique d'une migration définitive. Ce résultat va dans le sens de l'hypothèse $\mathrm{H} 3 \mathrm{a}$. II est possible que leur dernier séjour ait été une longue migration de transit destinée à atteindre l'Europe, ou que leur projet migratoire ait échoué dans le pays de destination précédent et qu'ils tentent à présent leur chance en Europe. Dans ces cas-là, ils tendent à ne pas penser à la possibilité de retourner vivre dans leur pays d'origine vu le coût élevé qu'ils ont investi dans leur migration.

Dans le même sens, les Congolais en situation irrégulière l'année de leur arrivée en Europe sont moins susceptibles d'avoir l'intention de retourner par rapport à ceux qui ont un statut légal. L'hypothèse $\mathrm{H} 2 \mathrm{a}$ est donc vérifiée pour les Congolais, mais pas pour les Sénégalais. Motivés par l'absence de perspectives d'avenir dans leur pays d'origine, les migrants congolais ont tout mis en œuvre pour migrer en Europe malgré le contexte politique migratoire restrictif. Leur migration a dû se révéler coûteuse, non seulement au niveau financier - car migrer sans papier est plus onéreux que migrer de façon régulière - mais aussi au niveau humain - car ils savent qu'ils encourent le risque de se faire expulser. Cela peut expliquer le fait qu'ils espèrent que ces risques pris leur permettront de ne pas devoir retourner dans leur pays d'origine, où ils n'envisagent pas leur avenir. 
Tableau 3. Déterminants de l'intention de retourner au pays par rapport à l'intention de rester en Europe pour les migrants congolais et sénégalais au moment de leur arrivée en Europe (régression logistique multinomiale ; résultats pondérés et exprimés sous forme de rapports de cotes) (a)

\begin{tabular}{|c|c|c|c|c|c|}
\hline & \multirow{2}{*}{\multicolumn{2}{|c|}{ Sénégal }} & & \\
\hline & & & & \multicolumn{2}{|c|}{ RD Congo } \\
\hline Variables & Modalités & Effets bruts & Effets nets & $\begin{array}{l}\text { Effets } \\
\text { bruts }\end{array}$ & $\begin{array}{c}\text { Effets } \\
\text { nets }\end{array}$ \\
\hline \multirow{3}{*}{ Age } & $18-29$ ans (réf) & 1 & 1 & 1 & 1 \\
\hline & $30-44$ ans & 1,31 & 1,47 & 2,00 & 1,90 \\
\hline & 45 ans et + & 1,62 & 2,11 & 0,37 & 1,66 \\
\hline \multirow{2}{*}{ Sexe } & Hommes (réf) & 1 & 1 & 1 & 1 \\
\hline & Femmes & 0,80 & $1,68^{*}$ & 0,61 & 0,75 \\
\hline \multirow{2}{*}{$\begin{array}{l}\text { Niveau } \\
\text { d'éducation } \\
\text { atteint }\end{array}$} & Primaire ou secondaire (réf) & 1 & 1 & 1 & 1 \\
\hline & Supérieur & $14,23 * *$ & 4,05 & 0,77 & 1,32 \\
\hline \multirow{4}{*}{$\begin{array}{l}\text { Motif de la } \\
\text { migration }\end{array}$} & Famille (réf) & 1 & 1 & 1 & 1 \\
\hline & $\begin{array}{l}\text { Amélioration des conditions } \\
\text { de vie }\end{array}$ & $2,19^{*}$ & $3,12 * *$ & 0,53 & 0,58 \\
\hline & $\begin{array}{l}\text { Etudes ou mission } \\
\text { professionnelle }\end{array}$ & $13,81^{* * *}$ & $12,55^{* * *}$ & $16,71^{* * *}$ & $13,56 * * *$ \\
\hline & Raison politique & $2,73^{* * *}$ & 0,47 & 0,88 & 1,57 \\
\hline \multirow{2}{*}{$\begin{array}{l}\text { Rang de la } \\
\text { migration }\end{array}$} & Première (réf) & 1 & 1 & 1 & 1 \\
\hline & Pas première & 1,49 & 1,22 & 1,40 & 0,90 \\
\hline \multirow{2}{*}{$\begin{array}{l}\text { Situation } \\
\text { administrative }\end{array}$} & En situation régulière (réf) & 1 & 1 & 1 & 1 \\
\hline & En situation irrégulière & 0,71 & 1,22 & $0,10^{* * *}$ & $0,42 * *$ \\
\hline \multirow{2}{*}{$\begin{array}{l}\text { Provenance } \\
\text { directe du pays } \\
\text { d'origine }\end{array}$} & Oui (réf) & 1 & 1 & 1 & 1 \\
\hline & Non & $0,34^{*}$ & $0,32 *$ & $0,10^{* * *}$ & $0,17^{* * *}$ \\
\hline \multirow[t]{2}{*}{ Destination } & $\begin{array}{l}\text { Ancienne / traditionnelle } \\
\text { (réf) }\end{array}$ & 1 & 1 & 1 & 1 \\
\hline & Nouvelle & $0,44^{* * *}$ & $0,44^{* * *}$ & $0,29 * * *$ & $0,19^{* * *}$ \\
\hline \multirow{4}{*}{$\begin{array}{l}\text { Situation } \\
\text { familiale }\end{array}$} & $\begin{array}{l}\text { Famille dans pays d'origine } \\
\text { (réf) }\end{array}$ & 1 & 1 & 1 & 1 \\
\hline & $\begin{array}{l}\text { Famille dans pays de } \\
\text { destination }\end{array}$ & 0,60 & 0,81 & 0,44 & 1,14 \\
\hline & Célibataire sans enfant & 0,94 & 0,87 & 0,50 & 0,50 \\
\hline & $\begin{array}{l}\text { Famille dans pays de } \\
\text { destination et d'origine }\end{array}$ & $0,37 * *$ & 0,46 & 0,54 & 0,89 \\
\hline \multirow{2}{*}{$\begin{array}{l}\text { Situation } \\
\text { matérielle }\end{array}$} & Propriétaire (réf) & 1 & 1 & 1 & 1 \\
\hline & Pas propriétaire & 0,72 & 0,97 & 2,63 & 1,88 \\
\hline \multirow{2}{*}{ Période d'arrivée } & Avant 1990 (réf) & 1 & 1 & 1 & 1 \\
\hline & Après 1990 & $0,56 * *$ & 0,70 & $0,24^{*}$ & $0,18^{* * *}$ \\
\hline Constante & & & 0,47 & & 2,64 \\
\hline $\begin{array}{l}\text { Nombre } \\
\text { d'événements }\end{array}$ & & \multicolumn{2}{|c|}{292} & \multicolumn{2}{|c|}{224} \\
\hline $\begin{array}{l}\text { Nombre } \\
\text { d'observations }\end{array}$ & & \multicolumn{2}{|c|}{713} & \multicolumn{2}{|c|}{521} \\
\hline
\end{tabular}

${ }^{* * *}: p<0.01 ; * *: p<0.05 ;^{*}: p<0.10$

Source : Enquêtes biographiques MAFE-Sénégal (2008) et MAFE-Congo (2009).

(a) Les estimations des rapports de cotes supérieures à 10 ou inférieures à 0,1 sont fragiles.

\section{La réalisation de l'intention initiale de retour}

Après avoir analysé les facteurs de l'intention initiale de retour, d'autres questions se posent. Premièrement, l'intention de retour influence-t-elle la réalisation du retour ? Les résultats des modèles (tableau 4) sont unanimes à ce propos : I'intention initiale de retour est un déterminant fort du retour. Les Sénégalais et les Congolais qui ont migré en Europe dans une optique temporaire ont respectivement deux et trois fois plus de chances de retourner que ceux qui entendaient $s^{\prime} y$ installer définitivement. Cependant, comme le tableau 4 le montre, ce n'est pas le seul déterminant. La période apparait aussi comme un facteur important du retour pour les migrants congolais. Ceux qui sont en Europe dans les années 1990 et 2000 sont moins susceptibles de retourner, ce qui s'explique par la 
détérioration de la situation dans leur pays d'origine. Les politiques migratoires devenues plus restrictives en Europe ont aussi un effet sur le retour. Les Congolais qui ne sont pas arrivés de façon directe en Europe n'ont pratiquement aucune chance de retourner (conformément à l'intention qu'ils avaient au moment de leur arrivée). Le fait de ne pas avoir atteint l'Europe directement leur a été coûteux en forces et en argent et ne les incite pas à retourner, car ils ne sont pas prêts à prendre le risque d'avoir à renouveler l'expérience. L'hypothèse $\mathrm{H} 2 \mathrm{~b}$ se vérifie donc pour les Congolais, mais pas pour les Sénégalais, ce qui peut s'expliquer par le contexte qui est relativement moins difficile au Sénégal. II est en de même pour l'hypothèse H3b. En effet, les Congolais en situation irrégulière ont de très faibles chances de retourner, et leur probabilité de rester en Europe est très importante (ce qui correspond à leur intention initiale). Ils ne retournent pas tant que leur situation n'a pas été régularisée, sans quoi ils savent qu'ils n'auront pas l'assurance de repartir en Europe en cas d'échec ou de problème après leur retour. Ainsi, le fait de pouvoir repartir en Europe après le retour conditionne le retour car cela permet aux migrants de gérer les incertitudes de la réinsertion.

Deuxièmement, quels sont les migrants qui ne réalisent pas un retour alors qu'ils en avaient initialement l'intention ? La comparaison de la variable sur le motif de la migration dans les tableaux 3 et 4 met en évidence des résultats qui confirment partiellement l'hypothèse $\mathrm{H} 1 \mathrm{~b}$. Les Congolais qui ont migré en Europe dans un cadre professionnel ou d'études, qui présentaient des chances importantes de vouloir retourner au moment de leur arrivée, ne sont finalement pas très susceptibles de retourner. Ils décident de rester plus durablement que prévu en Europe probablement parce qu'ils n'entrevoient pas des perspectives d'avenir durables en RD Congo. En revanche, la probabilité de retour pour les migrants sénégalais qui ont migré pour cette même raison est élevée. La situation plus favorable au Sénégal explique qu'ils réalisent leur intention initiale de retour car ils savent qu'ils y auront des opportunités professionnelles intéressantes. Ensuite, les Sénégalais partis pour améliorer leurs conditions de vie, qui étaient nombreux à avoir la volonté de retourner, n'ont finalement que de faibles chances d'effectuer un retour. Il est possible que les réalités de l'Europe ne correspondent pas à leurs attentes et que l'accumulation de capital financier soit plus ardue que prévu, ce qui explique qu'ils retardent leur retour. Quant aux Congolais qui ont migré pour des raisons politiques, ils retournent encore moins que ce qu'ils prévoyaient au début de leur migration. Cela signifie qu'une certaine rupture a lieu entre ces migrants et leur pays d'origine. En outre, l'effet de la situation familiale sur le retour révèle que les migrants qui vivent avec leur famille en Europe sont moins susceptibles de retourner que ceux dont la famille se trouve dans le pays d'origine, alors que ce n'était pas leur intention initiale. Avec le temps, la vie en famille en Europe donne donc lieu à une installation plus définitive que prévu. 
Tableau 4. Déterminants du retour au pays pour les Sénégalais et les Congolais qui ont migré en Europe (régression logistique en temps discret ; résultats pondérés et exprimés sous forme de rapports de cotes) (a)

\begin{tabular}{|c|c|c|c|c|c|}
\hline \multirow{2}{*}{ Variables } & \multirow[b]{2}{*}{ Modalités } & \multicolumn{2}{|l|}{ Sénégal } & \multicolumn{2}{|l|}{ RD Congo } \\
\hline & & Effets bruts & $\begin{array}{l}\text { Effets } \\
\text { nets }\end{array}$ & Effets bruts & $\begin{array}{l}\text { Effets } \\
\text { nets }\end{array}$ \\
\hline \multirow{3}{*}{ Age } & $18-29$ ans & 1 & 1 & 1 & 1 \\
\hline & $30-44$ ans & 1,07 & 1,21 & 0,63 & 1,06 \\
\hline & 45 ans et + & 0,66 & 1,11 & 0,85 & 2,93 \\
\hline \multirow{2}{*}{ Sexe } & Hommes (réf) & 1 & 1 & 1 & 1 \\
\hline & Femmes & 1,26 & 1,58 & 0,52 & 0,63 \\
\hline \multirow{2}{*}{$\begin{array}{l}\text { Niveau d'éducation } \\
\text { atteint }\end{array}$} & Aucun, primaire ou secondaire (réf) & 1 & 1 & 1 & 1 \\
\hline & Supérieur & 1,10 & 0,67 & 1,19 & 2,00 \\
\hline \multirow{4}{*}{ Motif de la migration } & Famille (réf) & 1 & 1 & 1 & 1 \\
\hline & Amélioration des conditions de vie & 0,66 & 0,81 & 1,19 & 1,41 \\
\hline & Etudes ou mission professionnelle & $2,78^{*}$ & $2,53^{\star}$ & $3,62^{* *}$ & 2,71 \\
\hline & Raison politique & 1 & 1 & $0,07^{\star \star \star}$ & $0,07^{\star \star}$ \\
\hline \multirow{2}{*}{ Rang de la migration } & Première (réf) & 1 & 1 & 1 & 1 \\
\hline & Pas première & $6,36^{\star * *}$ & $2,75^{\star \star}$ & 0,70 & 0,35 \\
\hline \multirow{3}{*}{ Durée } & $1-2$ ans (réf) & 1 & 1 & 1 & 1 \\
\hline & $3-5$ ans & 0,81 & $2,18^{\star \star}$ & 0,58 & 0,86 \\
\hline & 6 ans et + & $0,28^{\star \star \star}$ & 1,75 & $0,35^{\star \star}$ & 0,59 \\
\hline \multirow{2}{*}{ Visite(s) } & Jamais de visite (réf) & 1 & 1 & 1 & 1 \\
\hline & Au moins une visite & $0,14^{\star \star \star}$ & $0,16^{\star \star \star}$ & $0,80^{\star *}$ & 0,68 \\
\hline \multirow{3}{*}{$\begin{array}{l}\text { Intention initiale } \\
\text { quant au retour }\end{array}$} & Pas d'intention de retourner (réf) & 1 & 1 & 1 & 1 \\
\hline & Intention de retourner & $2,38^{* *}$ & $2,43^{\star \star \star}$ & $5,16^{\star * *}$ & $3,42^{*}$ \\
\hline & Incertitude & 0,70 & 1,04 & 5,76 & 15,16 \\
\hline \multirow{2}{*}{$\begin{array}{l}\text { Situation } \\
\text { administrative }\end{array}$} & En situation régulière (réf) & 1 & 1 & 1 & 1 \\
\hline & En situation irrégulière & 1,04 & 0,59 & $0,01^{\star \star \star}$ & $0,02^{\star \star \star}$ \\
\hline \multirow{2}{*}{$\begin{array}{l}\text { Provenance directe } \\
\text { du pays d'origine }\end{array}$} & Oui (réf) & 1 & 1 & 1 & 1 \\
\hline & Non & 2,43 & 0,84 & $0,04^{* * *}$ & $0,09^{*}$ \\
\hline \multirow{2}{*}{ Destination } & Ancienne / traditionnelle (réf) & 1 & 1 & 1 & 1 \\
\hline & Nouvelle & $0,56^{*}$ & 0,76 & 0,96 & 1,32 \\
\hline \multirow{5}{*}{ Situation familiale } & Famille dans pays d'origine (réf) & 1 & 1 & 1 & 1 \\
\hline & Famille dans pays de destination & $0,18^{\star \star \star}$ & $0,15^{\star \star \star}$ & $0,03^{\star \star \star}$ & $0,18^{\star \star \star}$ \\
\hline & Célibataire sans enfant & 0,76 & 0,67 & 0,65 & 1,47 \\
\hline & $\begin{array}{l}\text { Famille dans pays de destination et } \\
\text { d'origine }\end{array}$ & $0,33^{*}$ & $0,23^{\star}$ & 0,57 & 4,10 \\
\hline & Famille ailleurs & $8,51^{\star \star}$ & 3,75 & 1,00 & 1,00 \\
\hline \multirow{2}{*}{ Situation matérielle } & Propriétaire (réf) & 1 & 1 & 1 & 1 \\
\hline & Pas propriétaire & 0,80 & 0,58 & 1,53 & 0,93 \\
\hline \multirow{4}{*}{$\begin{array}{l}\text { Situation } \\
\text { professionnelle }\end{array}$} & $\begin{array}{l}\text { Actif à hauteur de ses compétences } \\
\text { (réf) }\end{array}$ & 1 & 1 & 1 & 1 \\
\hline & Actif en situation de sous-emploi & 1,31 & 1,09 & 2,27 & 4,64 \\
\hline & Inactif & $3,72^{\star \star}$ & $3,40^{\star \star}$ & $4,33^{\star \star \star}$ & $13,90^{\star \star}$ \\
\hline & Aux études & $2,82^{\star \star *}$ & 0,71 & $6,98^{\star * *}$ & 3,06 \\
\hline \multirow{2}{*}{$\begin{array}{l}\text { Situation économique } \\
\text { du ménage }\end{array}$} & Moyens plus que suffisants (réf) & 1 & 1 & 1 & 1 \\
\hline & Moyens pas plus que suffisants & $0,35^{\star \star}$ & 0,99 & $0,29^{\star \star \star}$ & $0,25^{\star \star}$ \\
\hline \multirow{2}{*}{ Période courante } & Avant 1990 (réf) & 1 & 1 & 1 & 1 \\
\hline & A partir de 1990 & $0,44^{\star \star \star}$ & 0,59 & $0,18^{\star \star \star}$ & $0,22^{\star \star \star}$ \\
\hline Constante & & & $0,03^{\star \star *}$ & & $0,01^{* *}$ \\
\hline $\begin{array}{l}\text { Nombre } \\
\text { d'événements }\end{array}$ & & \multicolumn{2}{|c|}{104} & \multicolumn{2}{|c|}{86} \\
\hline $\begin{array}{l}\text { Nombre } \\
\text { d'observations } \\
\text { (personnes-années) }\end{array}$ & & \multicolumn{2}{|c|}{8041} & & \\
\hline
\end{tabular}

${ }^{* * *}: \mathrm{p}<0.01 ;{ }^{* *}: \mathrm{p}<0.05 ;^{*}: \mathrm{p}<0.10$

Source : Enquêtes biographiques MAFE-Sénégal (2008) et MAFE-Congo (2009).

(a) Les estimations des rapports de cotes supérieures à 10 ou inférieures à 0,1 sont fragiles.

Troisièmement, on peut s'interroger sur les différences d'intentions initiales quant au retour selon la période. Les résultats descriptifs de la figure 2 montrent que la réalisation des intentions de retours est moins fréquente et moins rapide parmi les migrants arrivés après 1990 par rapport à ceux arrivés après cette date. Si, après 10 ans passés en Europe, $75 \%$ des Congolais qui ont migré avant 1990 en ayant l'intention de retourner ont effectué un retour, la proportion tombe à $40 \%$ parmi ceux arrivés avec cette même intention après 1990 . Du côté sénégalais, $40 \%$ des migrants arrivés avant 1990 sont retournés au Sénégal comme ils le 
prévoyaient après 10 ans, mais cette proportion n'est plus que de $25 \%$ pour ceux qui sont arrivés en Europe par la suite.

Figure 2. Evolution de la probabilité de rester en Europe en fonction de la durée du séjour et selon l'intention initiale quant au retour, par période d'arrivée (résultats pondérés) (a)

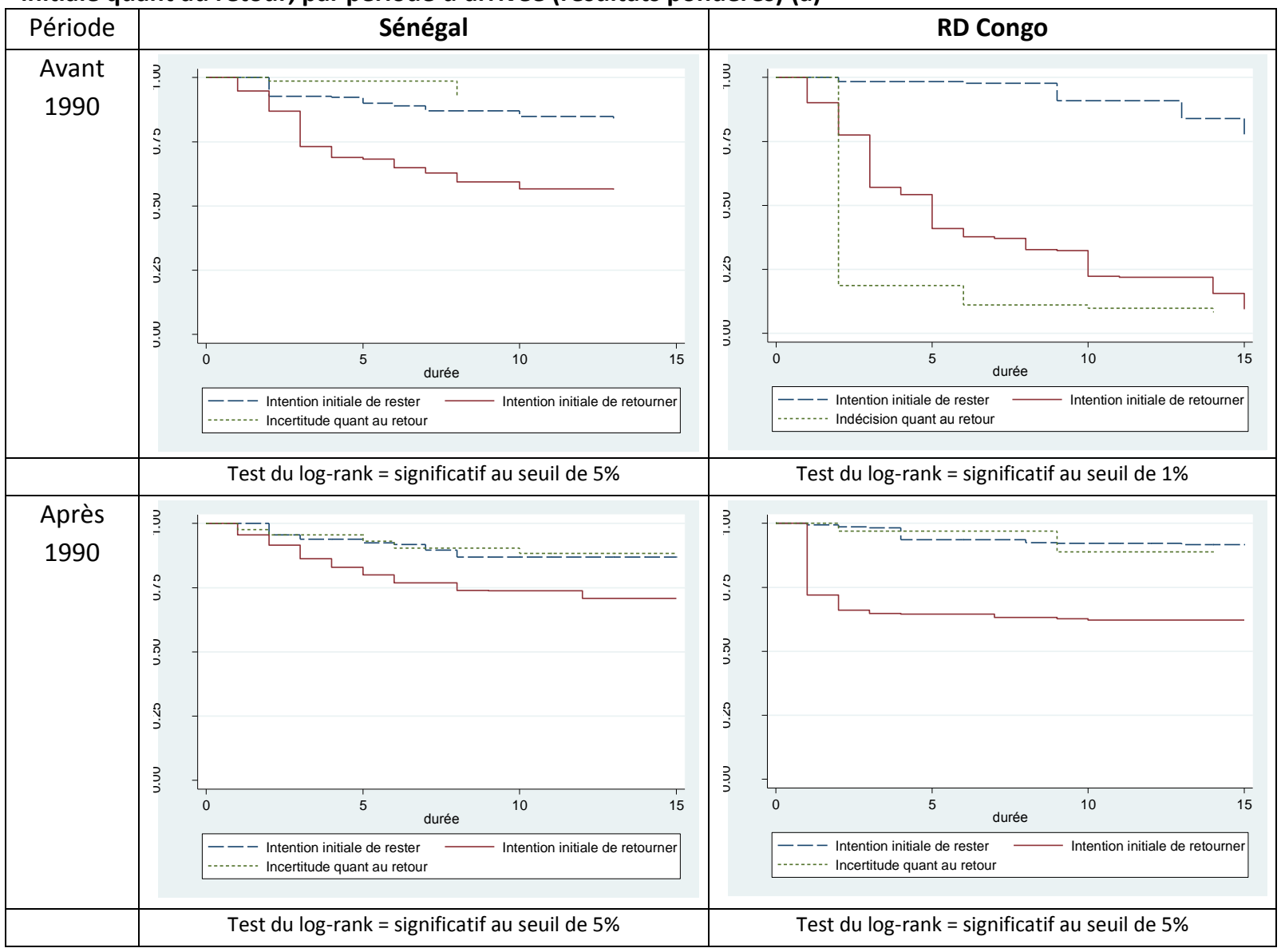

Source : Enquêtes biographiques MAFE-Sénégal (2008) et MAFE-Congo (2009).

(a) Lecture : Les courbes représentent les probabilités qu'ont les migrants de rester en Europe plutôt que de retourner, en fonction du nombre d'années écoulées depuis leur arrivée et selon leur intention initiale de retour. L'origine de l'axe des abscisses (année 0) correspond à l'année du départ. Cette année-là, 100\% (noté 1.00 sur l'axe des ordonnées) des migrants sont encore en Europe. A mesure que le temps passe, la probabilité d'y rester diminue, ce qui signifie - a contrario- que la probabilité d'effectuer un retour progresse. Ces courbes ont été construites en utilisant l'estimateur non-paramétrique de la fonction de Kaplan-Meier.

Cependant, toutes choses égales par ailleurs, les analyses du tableau 5 sur l'effet de l'interaction des variables " période " et "intention initiale quant au retour " (prenant en compte les mêmes covariables ${ }^{6}$ que dans le tableau 4) montrent que l'intention initiale de retour n'apparaît pas toujours comme un déterminant important du retour. Pour les migrants sénégalais, elle influençait les retours avant 1990, mais ne s'avère plus déterminante pour les migrants qui sont en Europe après 1990, ce qui confirme l'hypothèse $\mathrm{H} 4 \mathrm{~b}$. On peut rapprocher ce résultat aux conditions de vie devenues généralement plus difficiles au Sénégal après 1990, décourageant de cette façon les retours, même lorsque les migrants envisageaient de retourner au moment de leur arrivée en Europe. Les réalités économiques plus difficiles au Sénégal impliquent également que les besoins des familles restées au pays deviennent plus importants, ce qui engendre plus de charges pour les migrants dont la responsabilité est de subvenir aux besoins familiaux. De ce fait, ils restent plus longtemps que prévu en Europe afin d'y acquérir le capital financier nécessaire pour

\footnotetext{
${ }^{6}$ Les effets de ces covariables sont proches de ceux du tableau 4.
} 
leurs propres projets. En ce qui concerne les migrants congolais, toutes choses égales par ailleurs, ceux qui envisageaient un retour ne sont finalement pas plus susceptibles de retourner, ni avant, ni après 1990 (hypothèse $\mathrm{H} 4 \mathrm{~b}$ partiellement vérifiée). La profonde détérioration du contexte en RD Congo explique ce résultat. Même s'ils planifiaient un retour, les migrants ne prennent pas le risque de retourner car ils savent que la situation dans leur pays d'origine est instable et difficile.

Tableau 5. Effet de l'intention initiale des migrants quant au retour sur la réalisation du retour, par période, pour les Sénégalais et les Congolais qui ont migré en Europe (régression logistique en temps discret ; résultats pondérés et exprimés sous forme de rapports de cotes) (a)

\begin{tabular}{|c|c|c|c|c|c|c|c|c|c|}
\hline & & \multicolumn{4}{|c|}{ Sénégal } & \multicolumn{4}{|c|}{ DR Congo } \\
\hline Variable & Modalités & $\begin{array}{l}\text { Effets } \\
\text { bruts } \\
\text { (a) }\end{array}$ & $\begin{array}{l}\text { Effets } \\
\text { bruts } \\
\text { (b) }\end{array}$ & $\begin{array}{c}\text { Effets } \\
\text { nets } \\
\text { (a) }\end{array}$ & $\begin{array}{c}\text { Effets } \\
\text { nets } \\
\text { (b) }\end{array}$ & $\begin{array}{c}\text { Effets } \\
\text { bruts } \\
\text { (a) }\end{array}$ & $\begin{array}{l}\text { Effets } \\
\text { bruts } \\
\text { (b) }\end{array}$ & $\begin{array}{c}\text { Effets } \\
\text { nets } \\
\text { (a) }\end{array}$ & $\begin{array}{c}\text { Effets } \\
\text { nets } \\
\text { (b) }\end{array}$ \\
\hline \multirow{6}{*}{$\begin{array}{l}\text { Interaction } \\
\text { intention initiale } \\
\text { quant au retour } \\
\& \text { période } \\
\text { courante }\end{array}$} & $\begin{array}{l}\text { Avant } 1990 \\
\text { sans } \\
\text { intention de } \\
\text { retour } \\
\text { (réf a) }\end{array}$ & 1 & 1,08 & 1 & 0,92 & 1 & 1,48 & 1 & 3,18 \\
\hline & $\begin{array}{l}\text { Avant } 1990 \\
\text { avec } \\
\text { intention de } \\
\text { retour } \\
\end{array}$ & $4,83^{*}$ & 5,51 & $4,89 *$ & $4,51^{* * *}$ & $6,69 * *$ & $9,92^{* * *}$ & 4,85 & $15,44 * * *$ \\
\hline & $\begin{array}{l}\text { Avant } 1990 \\
\text { avec } \\
\text { incertitude }\end{array}$ & 0,52 & 0,56 & 0,85 & 0,79 & 18,40 & $27,30 *$ & $150,75^{* *}$ & $490,49 * *$ \\
\hline & $\begin{array}{l}\text { Après } 1990 \\
\text { sans } \\
\text { intention de } \\
\text { retour } \\
\text { (réf b) }\end{array}$ & 0,92 & 1 & 1,08 & 1 & 0,67 & 1 & 0,31 & 1 \\
\hline & $\begin{array}{l}\text { Après } 1990 \\
\text { avec } \\
\text { intention de } \\
\text { retour }\end{array}$ & 1,62 & 1,74 & 2,08 & 1,92 & 1,75 & 2,60 & 1,48 & 4,69 \\
\hline & $\begin{array}{l}\text { Après } 1990 \\
\text { avec } \\
\text { incertitude }\end{array}$ & 0,71 & 0,76 & 1,37 & 1,26 & 0,24 & 0,35 & 0,26 & 0,82 \\
\hline Constante & & & & $0,02^{* * *}$ & $0,02^{* * *}$ & & & $0,01^{* *}$ & $0,00^{* * *}$ \\
\hline $\begin{array}{l}\text { Nombre } \\
\text { d'événements }\end{array}$ & & \multicolumn{4}{|c|}{104} & \multicolumn{4}{|c|}{86} \\
\hline $\begin{array}{l}\text { Nombre } \\
\text { d'observqtions } \\
\text { (personnes- } \\
\text { années) }\end{array}$ & & \multicolumn{4}{|c|}{8041} & \multicolumn{4}{|c|}{5893} \\
\hline
\end{tabular}

Source : Enquêtes biographiques MAFE-Sénégal (2008) et MAFE-Congo (2009).

(a) Les estimations des rapports de cotes supérieures à 10 ou inférieures à 0,1 sont fragiles.

\section{Conclusion}

Dans une publication de 2011, de Haas et Fokkema soulignent que l'analyse de l'évolution des stratégies initiales des migrants dans le temps constitue un défi important pour les recherches futures. Ce sujet n'est pas développé dans les théories traditionnelles des migrations internationales et n'est pas abordé dans les études quantitatives existantes, faute de données appropriées. Grâce aux enquêtes biographiques du projet MAFE, qui ont interrogé des migrants pendant leur séjour en Europe et des migrants de retour dans leur pays d'origine, cet article a apporté des éléments nouveaux sur les intentions initiales de retour des migrants sénégalais et congolais ainsi que sur la concrétisation de leurs intentions. Rappelons que l'information sur l'intention initiale de retour a été obtenue 
rétrospectivement, et que l'on ne dispose pas d'information précise sur le niveau d'incertitude dans lequel évoluent les migrants, ce qui pourrait faire l'objet de recherches approfondies dans le futur.

Cette recherche a révélé que l'intention de retour des migrants, au moment de leur arrivée en Europe, est liée à ce qu'ils estiment être le mieux pour eux et pour leur famille. Ainsi, ceux qui partent dans un cadre professionnel ou d'études sont généralement dans l'optique de retourner dans leur pays d'origine car ils savent que leur expérience migratoire aura un effet positif sur leur vie après le retour. Cependant, le projet migratoire initial des individus dépend aussi du contexte qui prévaut dans leur pays d'origine et au niveau des politiques migratoires. Lorsque les migrants quittent un pays en crise comme c'est le cas de la RD Congo depuis les années 1990, ils ont moins l'intention de retourner. De plus, ceux qui ont eu des difficultés à migrer en raison des politiques migratoires restrictives envisagent davantage de rester définitivement en Europe, car ils savent que, s'ils retournent, il leur sera difficile de migrer à nouveau par la suite, en cas d'échec pour se réinsérer ou de crise.

Les analyses ont également montré que, toutes périodes confondues, l'intention initiale de retour influence généralement la réalisation du retour, mais que certains migrants annulent ou remettent à plus tard le retour. L'intention initiale de retour ne s'avère toutefois plus déterminante pour les migrants qui sont en Europe dans les années 1990 et 2000 . Avec la détérioration du contexte au Sénégal et en RD Congo et les politiques migratoires restrictives, quand bien même ils ont l'intention de retourner au début de leur migration, celle-ci ne donne plus autant lieu à des retours. Pour que l'intention de retour des migrants se réalise, il faut donc que la situation dans leur pays d'origine n'hypothèque pas les perspectives de réinsertion, que les migrants aient acquis les ressources nécessaires à leur réinsertion, et que le contexte politique international facilite pour eux la gestion des incertitudes de la réinsertion, en leur permettant de circuler plus librement entre l'Afrique et l'Europe.

\section{Remerciements}

Je remercie Bruno Schoumaker et Cris Beauchemin pour leurs commentaires tout au long de ma recherche ainsi que les relecteurs anonymes pour leurs suggestions sur les versions précédentes de cet article. Je suis également reconnaissante envers le Fonds national de la recherche scientifique (F.R.S.-FNRS) et le Conseil européen de la recherche (dans le cadre du projet DEMIG - ERC Grant Agreement 240940) pour leur soutien financier.

\section{Bibliographie}

AZOULAY Muriel, QUIMINAL Catherine, 2002, "Reconstruction des rapports de genre en situation migratoire. Femmes réveillées, hommes menacés en milieu soninké », VEI Enjeux, 128, p. 87-101.

BAROU Jacques, 2001, "La famille à distance: nouvelles stratégies familiales chez les immigrés d'Afrique Sahélienne ", Hommes et Migrations, 1232, p. 16-25.

BARUCH Yehuda, BUDHWAR Pawan S., KHATRI Naresh, 2007, "Brain drain: Inclination to stay abroad after studies », Journal of World Business, 42 (1), p. 99-112.

BAZONZI José M., 2010, "Comprendre la mobilité féminine et la dynamique migratoire intraafricaine à partir du courant centrifuge au départ de Kinshasa ", Revue congolaise d'économie, 5 (1), p. 2-19.

BIJWAARD Govert E., 2007, "Modelling Migration Dynamics of Immigrants: The Case of the Netherlands ", IZA Discussion Paper, 2891, 46 p. 
BILGILI Özge, SIEGEL Melissa, 2012, « Migrant's Integration, Homeland Engagement and Intentions to Return. An Analysis based on Afghan, Burundian, Ethiopian and Moroccan Migrants in the Netherlands", communication présentée à l'atelier "Return migration and transnationalism: alternatives or complements" 4-5 septembre 2012, Peace Research Institute (PRIO), Oslo.

BOCQUIER Philippe, 1996, Insertion et mobilité professionnelles à Dakar, Etudes et Thèses, Paris, ORSTOM, $316 \mathrm{p}$.

BONGO-PASI MOKE SANGOL Willy, TSAKALA MUNIKENGI Télesphore, 2004, "Réinventer I'Université ou le paradoxe du diplôme à l'Université de Kinshasa ", in Trefon Théodore (dir.), Ordre et Désordre à Kinshasa. Réponses Populaires à La Faillite de l'Etat, Cahiers africains, Tervuren, Musée royal de l'Afrique centrale et L'Harmattan, p. 99-118.

BRAECKMAN Colette, 2009, Les nouveaux prédateurs : Politique des puissances en Afrique centrale, Bruxelles, Editions Aden, $394 \mathrm{p}$.

CARLING Jørgen, 2004, «Emigration, return and development in Cape Verde: the impact of closing borders", Population, Space and Place, 10 (2), p. 113-132.

CARLING Jørgen, MORTENSEN Elin B., Wu Jennifer, 2011, "A Systematic Bibliography on Return Migration ", PRIO Paper, Oslo, PRIO, $287 \mathrm{p}$.

CARLING Jørgen, HERNANDEZ-CARRETERO Maria, 2011, «Protecting Europe and Protecting Migrants? Strategies for Managing Unauthorised Migration from Africa », British Journal of Politics and International Relations, 13 (1), p. 42-58.

CARLING Jørgen, PETTERSEN Silje Vatne, 2014, "Return migration intentions in the integrationtransnationalism matrix », International Migration, DOI: 10.1111/imig.12161.

CASSARINO Jean-Pierre, 2004, "Theorising Return Migration: the Conceptual Approach to Return Migrants Revisited », International Journal on Multicultural Societies, 6 (2), p. 253-279.

CASSARINO Jean-Pierre, 2008, "The Conditions of Modern Return Migrants - Editorial Introduction ", International Journal on Multicultural Societies, 10 (2), p. 95-105.

CASTLES Stephen, 2006, "Guestworkers in Europe: A Resurrection? », International Migration Review, 40 (4), p. 741-766.

CONSTANT Amélie, MASSEY Douglas S., 2002, "Return Migration by German Guestworkers: Neoclassical versus New Economic Theories », International Migration, 40 (4), p. 5-38.

CORNELIUS Wayne A., 2001, "Death at the Border: Efficacy and Unintended Consequences of US Immigration Control Policy ", Population and Development Review, 27 (4), p. 661-685.

DE HAAS Hein, FOKKEMA Tineke, 2010, « Intra-Household Conflicts in Migration Decisionmaking: Return and Pendulum Migration in Morocco ", Population and Development Review, 36 (2), p. 541561.

DE HAAS Hein, FOKKEMA Tineke, 2011, "The effects of integration and transnational ties on international return migration intentions ", Demographic Research, 25, p. 755-782.

DE SCHUTTER Sam, 2011, "De "Anciens de Belgique" in de Democratische Republiek Congo. Een historisch onderzoek naar de impact van Belgische studies in de Congolese samenleving ", Mémoire de Master, Gand, Université de Gand, 138 p.

DONOVON Valentin, 1988, "Réalités françaises et conventions franco-africaines de circulation des personnes ", Peuples Noirs Peuples Africains, 59 (62), p. 149-164.

DUMONT Gérard-François., KANTE Seydou, 2009, "Le Sénégal : une géopolitique exceptionnelle en Afrique ", Géostratégiques, 10 (25), p. 107-133.

EDIN Per-Anders, LALONDE Robert J., ASLUND Olof, 2000, « Emigration of immigrants and measures of immigrant assimilation: Evidence from Sweden », Swedish economic policy review, 7, p. 163-204.

EL HARIRI Saâdia, 2003, "Les femmes et le retour au pays d'origine ", Hommes et migrations, 1242, p. 43-52.

FINOTELLI Claudia, SCIORTINO Giuseppe, 2009, "The Importance of Being Southern: The Making of Policies of Immigration Control in Italy », European Journal of Migration and Law, 11, p. 119-138. 
FLAHAUX Marie-Laurence, 2013, "Retourner au Sénégal et en RD Congo. Choix et contraintes au cœur des trajectoires de vie des migrants ", Louvain-la-Neuve, Presses universitaires de Louvain, 336 p.

GEERT Tiri, 2011, "Visa Policy as a Migration Channel in Europe ", European Migration Network, Belgian National Contact Point, Bruxelles, $99 \mathrm{p}$.

GNISCI Donata, 2008, West African mobility and migration policies of OECD countries, Paris, Organisation for Economic Co-operation and Development et Sahel and West Africa Club, 143 p.

GUILMOTO Christophe Z., SANDRON Frédéric, 2000, « La dynamique interne des réseaux migratoires dans les pays en développement », Population-F, 55 (1), p. 105-135.

GUIRAUDON Virginie, 2000, Les politiques d'immigration en Europe : Allemagne, France, Pays-Bas, Paris, L'Harmattan, $277 \mathrm{p}$.

HAZEN Helen D., ALBERTS Heike C., 2006, "Visitors or immigrants? International students in the United States », Population, Space and Place, 12 (3), p. 201-216.

HERNANDEZ-CARRETERO Maria, 2012, " Morals and belonging in Senegalese transnational dynamics and trajectories", Communication présentée à l'atelier "Return migration and transnationalism: alternatives or complements", 4-5 septembre 2012, Oslo, Peace Research Institute (PRIO), 15 p.

HESSELBEIN Gabi, 2007, "The rise and decline of the Congolese State. An analytical narrative of state-making ", Working Paper of the Crisis States Research Centre, London School of Economics, 21 p.

KAGNE Bonaventure, MARTINIELLO Marco, 2001, "L'immigration subsaharienne en Belgique ", Courrier hebdomadaire du CRISP, 1721 (16), p. 5-49.

MANGALU José A., 2011, " Migrations internationales, transfert des migrants et conditions de vie des ménages d'origine. Cas de la ville de Kinshasa ", Louvain-la-Neuve, Presses Universitaires de Louvain, $310 \mathrm{p}$.

MAROT Nadia, 1995, "L'évolution des accords franco-africains », Plein droit, 29-30, p. 96-99.

MASSEY Douglas S., ALARCON Rafael, DURAND Jorge, GONZALEZ Humberto, 1987, Return to Aztlan The Social Process of International Migration from Western Mexico, Berkeley, University of California Press, Studies in Demography, $332 \mathrm{p}$.

MASSEY Douglas S., DURAND Jorge, MALONE Nolan J., 2002, Beyond smoke and mirrors: Mexican immigration in an era of economic integration, New York, Russell Sage Foundation, $197 \mathrm{p}$.

MASSEY Douglas S., ESPINOSA Kristin, 1997, "What's Driving Mexico-U.S. Migration? A Theoretical, Empirical, and Policy Analysis », American Journal of Sociology, 102 (4), p. 939-999.

PEEMANS Jean-Philippe, 1998, Le Congo-Zaïre au gré du XXe siècle. État, économie, société 18801990, Paris, L'Harmattan, $279 \mathrm{p}$.

REYES Belinda, 2004, "Changes in Trip Duration for Mexican Immigrants to the United States", Population Research and Policy Review, 23 (3), p. 235-257.

ROBIN Nelly, LALOU Richard, NDIAYE Mamadou, 2000, Facteurs d'attraction et de répulsion à l'origine des flux migratoires internationaux : rapport national Sénégal, Dakar, Eurostat, $174 \mathrm{p}$.

SAYAD Abdelmalek, 2008, "Le retour, élément constitutif de la condition de l'immigré ", Migrations Société, 10 (57), p. 9-45.

SCHOONVAERE Quentin, 2010, Etude de la migration congolaise et de son impact sur la présence congolaise en Belgique. Analyse des principales données démographiques, Bruxelles, Centre pour l'égalité des chances et la lutte contre le racisme, $87 \mathrm{p}$.

SINATTI Giulia, 2011, "Mobile transmigrants" or "unsettled returnees"? Myth of return and permanent resettlement among Senegalese migrants ", Population, Space and Place, 17 (2), p. 153166.

STARK Oded, BLOOM David, 1985, "The New Economics of Labor Migration ", The American Economic Review, 75 (2), p. 173-178.

SUMATA Claude, TREFON Théodore, COGELS Serge, 2004, "Images et usages de l'argent de la diaspora congolaise : Les transferts comme vecteur d'entretien du quotidien à Kinshasa ", in Trefon 
Théodore (dir.), Ordre et Désordre à Kinshasa. Réponses Populaires à La Faillite de l'État, Cahiers Africains, Tervuren, Musée royal de l'Afrique centrale et L'Harmattan, p. 134-154.

THIOUB Ibrahima, DIOP Momar-Coumba, BOONE Catherine, 1998, "Economic Liberalization in Senegal: Shifting Politics of Indigenous Business Interests ", African Studies Review, 41 (2), p. 63-89.

TIMERA Mahamed, 1996, Les Soninké en France, Paris, Karthala, $250 \mathrm{p}$.

TODARO, Michael P., 1969, "A model of Labor Migration and Urban Unemployment in Less Developed Countries », American Economic Review, 59 (1), p. 138-148.

\section{Annexes}

Annexe 1. Explications de certaines variables explicatives.

- Niveau d'éducation atteint: Distinction entre les migrants ayant étudié au moins 3 années dans l'enseignement supérieur et ceux qui n'ont pas atteint ce niveau.

- Rang de la migration : Distinction entre les migrants effectuant une première migration en Europe et ceux qui ont déjà migré en Europe auparavant.

- Visite(s) : Distinction entre les migrants n'ayant jamais effectué une visite de moins d'un an dans leur pays d'origine dans un cadre familial ou professionnel et ceux qui ont déjà effectué un tel séjour.

- Situation administrative: Distinction entre les migrants qui ont des papiers (visa, titre de séjour, autre document de ce type) ou qui n'en ont pas besoin et ceux ne disposant d'aucun statut légal.

- Provenance directe du pays d'origine: Distinction entre les migrants qui ont vécu pendant au moins une année dans un pays autre que le leur avant d'arriver en Europe et ceux qui sont arrivés directement en Europe.

- Destination: Distinction entre les pays de destination "anciens " ou "traditionnels " (France pour les Sénégalais ; Belgique pour les Congolais) et les autres pays de destination.

- Situation familiale : Distinction entre les migrants dont la famille (conjoint et enfants) se trouve dans le pays d'origine, dans le pays de destination, entre ces deux pays, ailleurs, ou qui sont célibataires sans enfant.

- Situation matérielle: Distinction entre les migrants qui sont propriétaires d'un bien immobilier ou d'une affaire dans le pays d'origine ou de destination et ceux qui n'ont pas de propriété.

- Situation professionnelle : Distinction entre les migrants travaillant à hauteur de leur niveau de compétences, en-dessous de leur niveau de compétences, ceux qui sont inactifs, et les étudiants.

- Situation économique du ménage: Distinction entre les individus vivant en Europe dans des ménages à propos desquels ils déclarent que les ressources dont ils disposent pour vivre au quotidien sont plus que suffisantes, et ceux qui vivent dans des ménages dans lesquels les ressources sont tout juste suffisantes ou insuffisantes. 
Annexe 2. Déterminants de l'incertitude de retourner au pays par rapport à l'intention de rester en Europe pour les migrants congolais et sénégalais au moment de leur arrivée en Europe (suite de la régression logistique multinomiale du tableau 3 ; résultats pondérés et exprimés sous forme de rapports de cotes) (a)

\begin{tabular}{|c|c|c|c|c|c|}
\hline \multirow[b]{2}{*}{ Variables } & \multirow[b]{2}{*}{ Modalités } & \multicolumn{2}{|c|}{ Sénégal } & \multicolumn{2}{|c|}{ RD Congo } \\
\hline & & Effets bruts & Effets nets & Effets bruts & Effets nets \\
\hline \multirow{3}{*}{ Age } & 18-29 ans (réf) & 1 & 1 & 1 & 1 \\
\hline & $30-44$ ans & 0,99 & 1,15 & 1,05 & 1,24 \\
\hline & 45 ans et + & $4,14^{*}$ & 2,83 & 0,61 & 4,19 \\
\hline \multirow{2}{*}{ Sexe } & Hommes (réf) & 1 & 1 & 1 & 1 \\
\hline & Femmes & $2,04 *$ & $3,08 * * *$ & 0,39 & 0,24 \\
\hline \multirow{2}{*}{$\begin{array}{l}\text { Niveau } \\
\text { d'éducation } \\
\text { atteint }\end{array}$} & Primaire ou secondaire (réf) & 1 & 1 & 1 & 1 \\
\hline & Supérieur & 2,68 & $2,80 * * *$ & 0,58 & 1,01 \\
\hline \multirow{4}{*}{$\begin{array}{l}\text { Motif de la } \\
\text { migration }\end{array}$} & Famille (réf) & 1 & 1 & 1 & 1 \\
\hline & $\begin{array}{l}\text { Amélioration des conditions } \\
\text { de vie }\end{array}$ & 0,89 & $5,49 * * *$ & 0,75 & 0,79 \\
\hline & $\begin{array}{l}\text { Etudes ou mission } \\
\text { professionnelle }\end{array}$ & 2,13 & $4,93 * *$ & 13,67 & 7,00 \\
\hline & Raison politique & $2,00^{*}$ & $4,24 * * *$ & 2,56 & 2,98 \\
\hline \multirow{2}{*}{$\begin{array}{l}\text { Rang de la } \\
\text { migration }\end{array}$} & Première (réf) & 1 & 1 & 1 & 1 \\
\hline & Pas première & 0,69 & 0,94 & 1,01 & 0,70 \\
\hline \multirow{2}{*}{$\begin{array}{l}\text { Situation } \\
\text { administrative }\end{array}$} & En situation régulière (réf) & 1 & 1 & 1 & 1 \\
\hline & En situation irrégulière & 0,79 & 1,12 & 0,48 & 1,47 \\
\hline \multirow{2}{*}{$\begin{array}{l}\text { Provenance } \\
\text { directe du pays } \\
\text { d'origine }\end{array}$} & Oui (réf) & 1 & 1 & 1 & 1 \\
\hline & Non & 0,18 & 0,20 & 0,21 & $0,20 *$ \\
\hline \multirow{2}{*}{ Destination } & Ancienne / traditionnelle (réf) & 1 & 1 & 1 & 1 \\
\hline & Nouvelle & $0,17^{* * *}$ & $0,13^{* * *}$ & $0,03 * * *$ & $0,01 * * *$ \\
\hline \multirow{4}{*}{$\begin{array}{l}\text { Situation } \\
\text { familiale }\end{array}$} & $\begin{array}{l}\text { Famille dans pays d'origine } \\
\text { (réf) }\end{array}$ & 1 & 1 & 1 & 1 \\
\hline & $\begin{array}{l}\text { Famille dans pays de } \\
\text { destination }\end{array}$ & $2,28^{*}$ & 2,02 & 0,89 & 3,48 \\
\hline & Célibataire sans enfant & 1,18 & 1,13 & 1,97 & 2,58 \\
\hline & $\begin{array}{l}\text { Famille dans pays de } \\
\text { destination et d'origine }\end{array}$ & $2,98 * *$ & 2,02 & 0,97 & 2,20 \\
\hline \multirow{2}{*}{$\begin{array}{l}\text { Situation } \\
\text { matérielle }\end{array}$} & Propriétaire (réf) & 1 & 1 & 1 & 1 \\
\hline & Pas propriétaire & 0,84 & 0,89 & 2,95 & 2,46 \\
\hline \multirow{2}{*}{$\begin{array}{l}\text { Période } \\
\text { d'arrivée }\end{array}$} & Avant 1990 (réf) & 1 & 1 & 1 & 1 \\
\hline & Après 1990 & $0,46 * *$ & 0,72 & 0,26 & 0,11 \\
\hline Constante & & & $0,08 * * *$ & & 0,73 \\
\hline $\begin{array}{l}\text { Nombre } \\
\text { d'événements }\end{array}$ & & \multicolumn{2}{|c|}{61} & \multicolumn{2}{|c|}{44} \\
\hline $\begin{array}{l}\text { Nombre } \\
\text { d'observations }\end{array}$ & & \multicolumn{2}{|c|}{713} & \multicolumn{2}{|c|}{521} \\
\hline
\end{tabular}

***: $p<0.01 ;{ }^{* *}: p<0.05 ;{ }^{*}: p<0.10$

Source : Enquêtes biographiques MAFE-Sénégal (2008) et MAFE-Congo (2009).

(a) Les estimations des rapports de cotes supérieures à 10 ou inférieures à 0,1 sont fragiles. 